\title{
A MAC Protocol Based on Broadcast Messages for Wireless Sensor Network
}

\author{
Bingwu Kang ${ }^{1}$, Guoqiang Zheng ${ }^{1, *}$, Fangge $\mathrm{Nie}^{1}$, Huahong $\mathrm{Ma}^{1}$, Jishun $\mathrm{Li}^{2}$, Yujun Xue \\ and Peipei $\mathrm{Li}^{1}$ \\ ${ }^{I}$ School of Electronic Information Engineering, Henan University of Science and \\ Technology, Luoyang, 471003, China \\ ${ }^{2}$ Henan Key Laboratory for Machinery Design and Transmission System, Henan \\ University of Science and Technology, Luoyang, 471003, China \\ Corresponding Author: lyzhenggq@126.com
}

\begin{abstract}
Competitive MAC protocols become a good choice for Wireless Sensor Network because of their have good dynamic adaptability and so on. However, in general, there is a serious problem of data collision in competitive protocols, so a low collision MAC protocol based on broadcast messages is proposed in this paper, SA-MAC (Self-adaption Advertisement MAC). It uses broadcast messages to make the related nodes working in a scheduled time slot, while the unrelated nodes turning to sleep in the time slot, thus reducing the probability of data collision. Additionally, it designs the corresponding adaptive contention window mechanism and fast transmission mechanism according to the usage ratio of data buffer, so the network performance is further improved. The simulation results show that, compared with S-MAC and ADV-MAC, SA-MAC can reduce the energy consumption and delay while improving the packet delivery ratio. Moreover, it maintains a good network performance whether in the single-hop or multi-hop network environment.
\end{abstract}

Keywords: MAC, broadcast messages, SA-MAC, low collision, adaption

\section{Introduction}

Wireless Sensor Network (WSN) is a multi-hop and self-organizing network system that is composed of a large number of tiny sensor nodes, deployed in the surveillance area, which is formed through wireless communication [1].

Media Access Control (MAC) layer directly controls nodes to access the channel for data exchange, which has a decisive influence on WSN network performance and upper layer protocols. So, it is a good way to improve WSN network performance by improving MAC protocol to optimize the communication module [2].

At present, a large number of MAC protocols are proposed, which can be divided into scheduling class, competitive class and hybrid class according to the channel access mode $[3,4]$. Scheduling MAC protocols generally use TDMA mechanism, which has the following characteristics, the continuous time is divided into a series of consecutive time slots, nodes are awakened only in scheduled time slot to send and receive data packets [5]. TRAMA is a traffic-adaptive scheduling MAC protocol. It can achieve conflict-free communication in scheduled time slots which is determined by the actual network traffic flow. The nodes without communication turn to sleep mode, thus reducing the data collision and energy consumption due to the idle listening [6].Competitive MAC protocols generally use CSMA mechanism [7-8]. S-MAC is a typical competitive MAC protocol, which reduces idle listening by using the duty cycle mechanism, eliminates overhearing and guarantee the data integrity by using RTS/CTS/DATE/ACK data exchange mechanism, reduces the delay of multi-hop transmission by using the adaptive 
listening [9-10]. Hybrid MAC protocols are usually combined with different channel access methods for the MAC protocol. The typical representatives include Z-MAC and IMAC which use CSMA/TDMA mechanism [11-12], SMACS/EAR protocol which uses TDMA/FDMA mechanism [13].

Competitive MAC protocols are a better choice for WSN than others because of their good scalability, simple operation and low network cost. However, they have a serious problem of data collision due to the existence of hidden nodes, which seriously affects network performance.

On the basis of S-MAC, we propose a new competitive MAC protocol which is based on broadcast messages, SA-MAC (Self-adaption Advertisement MAC). The main objective is to reduce probability of data collision and idle listening time without compromising network performance. The senders use broadcast messages to make unrelated nodes turn to sleep, while the sender and the corresponding receiver are awakened in the scheduled time slot for data exchange [14]. SA-MAC is similar to ADVMAC which is proposed by S. Ray et al. [15-16]. However, SA-MAC has essentially different with ADV-MAC, which use broadcast messages to make unrelated nodes turning to sleep during network data exchange, while the related nodes still need to bear an intense channel competition.

\section{SA-MAC Design Overview}

SA-MAC is a MAC protocol with periodic wake mechanism. Similar to S-MAC, the virtual cluster is firstly formed by the exchange of the synchronous frame as shown in Figure 1. Some nodes are in the boundary region of different virtual clusters, which are defined as edge node. Such nodes need to consume more energy as a bridge between different virtual clusters. Therefore, we rule that such nodes do not implement data-aware task.

After the network initialization is completed, nodes enter into the periodic work phase, which includes synchronization phase, broadcast phase and data phase, as shown in Figure 2.

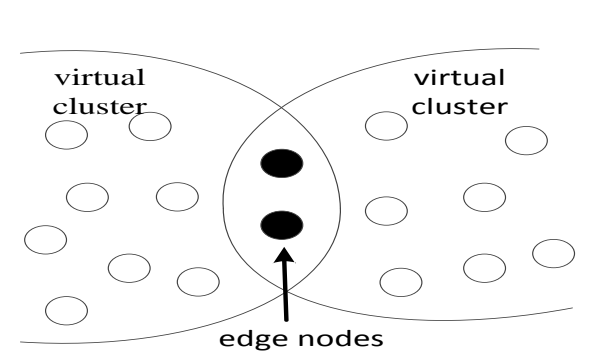

Figure 1. The Virtual Clusters

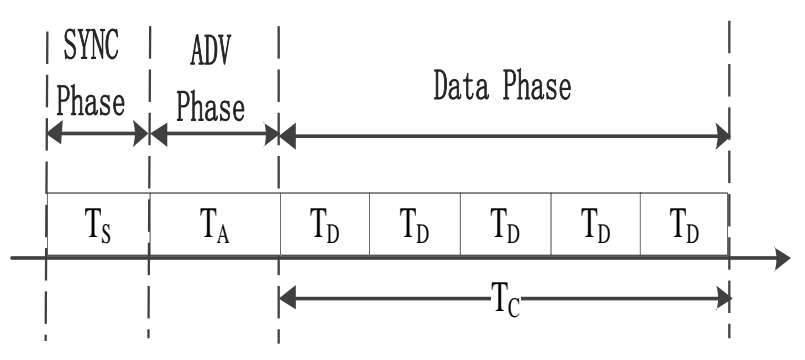

Figure 2. The Periodic Work

The time length of the synchronization phase is $T_{S}$, and its setting is similar to S-MAC. All nodes need to broadcast their own scheduling information regularly.

The total number of time slots is $m$, which the value is pre-setted by the protocol according to the service types and density of network nodes. The time length of the broadcast phase is $T_{A}$, which is determined by $m$. SA-MAC numbers the time slots from the beginning of one, and the time slot number ranges between $[1, m]$.

The senders complete the slot reservation in this phase by using the broadcast messages, which include the address of the receiver and the selected time slot number. Considering the network light load, nodes in the network will appear the phenomenon of continuous idle listening. So, we define a threshold Thadv, which is the maximum the time length during sending broadcast messages. In the case of the network light load, the time length of idle listening of the nodes without data packets sending task is only Thadv. 
The data phase is divided into a series of same length of time slots $T_{D}$, which is the longest time of nodes from the channel competition to a successfully complete RTS/CTS/DATE/ACK data exchange in the ideal condition, which is considered no data collision and the channel is good.

The time length $T_{C}$ of data phase is equal to $m^{*} T_{D}$. The nodes need to exchange data will be awaken in the scheduled time slot to complete data exchange.

The nodes use no acknowledgment mechanism to schedule time slot in the broadcast phase. So the protocol sets RTS waiting time Thdata, which is the maximum time of a node from the channel competition to send RTS in the ideal condition and sets CTS waiting time $T W C$, which is the time of nodes sending CTS in the ideal condition. If it appears the fading channel or data collision, the idle listening time of receivers is only Thdata, and the senders' time is only TWC after having sent RTS.

SA-MAC mainly includes two phases, broadcast phase and data phase. The following part will describe the implementation process of both the two phases respectively.

\subsection{Broadcast Phase}

SA-MAC rules that the channel reservation for nodes is only in the broadcast phase of each period, as long as there are nodes required to send the data can launch the channel reservation.

Considering that applications for WSN usually are uncertain, so we design a corresponding time slot selection algorithm. Its pseudo-code is shown in algorithm 1. All nodes maintain a random number generator, including three variables, the maximum time slot number NBmax which is equal to m, the minimum time slot number NBmin which is equal to 1 and the current maximum time slot number $N B$ cur. The senders will generate a random number $N B$, a randomly selected time slot number, which value ranges between [NBmin,NBcur], then compete channel to send broadcast messages. After current data exchange is completed, the nodes judge the communication result. If the data packet transmission failed, then $N B c u r$ is equal to $N B c u r+1$, otherwise $N B c u r$ is equal to $N B c u r-1$, the value of $N B c u r$ must range between [NBmin,NBmax].

\begin{tabular}{l}
\hline \multicolumn{1}{c}{ Algorithm 1. Time Slot Selection Algorithm } \\
\hline Step1: NBcur=[NBmax/2] (interger) \\
Step2: Need Send Data \\
Step3: NB=rand (NBmin,NBcur) \\
Step4: Observe The Situation Of Every Data Transmission \\
Step5: If Data Success \\
Step6: NBcur=NBcur-1 \\
Step7: If Data Fail \\
Step8: NBcur=NBcurr +1
\end{tabular}

The senders contend channel to send broadcast messages, because the different nodes have the different network load, therefore the priority for nodes to access channel should be different. For CSMA/CA mechanism, the probability of nodes access channel is mainly affected by the size of the contention window (CW). SA-MAC uses the node's data buffer (data queue) to dynamically adjust the size of $\mathrm{CW}$ in real-time, so as to achieve the fairness of the nodes access channel.

The pseudo-code of CW adaptation algorithm is shown in algorithm 2, wherein BST is a data buffer threshold which is decided by the network bandwidth and the capacity of data buffer. $B S$ is the usage of current data buffer, $C W c u r$ is the $\mathrm{CW}$ current value, $C W$ max is the maximum $\mathrm{CW}$ value, and $C W$ min is the minimum $\mathrm{CW}$ value. When nodes need to contend channel, the nodes use the formula $C W c u r=[C W \max (1-(B S / B S T))]$ 
(interger) to adjust the size of $C W c u r$ in real-time, the value of $C W c$ ur must range between [CWmin, CWmax].

\begin{tabular}{l}
\hline \multicolumn{1}{c}{ Algorithm 2. CW Adaptation Algorithm } \\
\hline Step1: Need Send Data And Observe Data Buffer \\
Step2: $C W$ cur $=[C W \max (1-(B S / B S T))]($ interger $)$ \\
Step3: if $C W c u r>=C W \max$ \\
Step4: $C W$ cur $=C$ wmax \\
Step5: if $C W$ cur $<=C W \min$ \\
Step6: $C W$ cur $=C W$ min \\
\hline
\end{tabular}

The probability of access channel will be affected by the usage of data buffer when nodes use adjusted $\mathrm{CW}$, it said, the more data packets in data buffer, the more easily access channel. Due to the limited time length in the broadcast phase, the nodes do not reset their back-off timer but temporarily freeze the timer, and waiting for channel is idle, if they have been listened the broadcast messages sent by other nodes. They continue to contending channel until the broadcast phase is over.

Typically, the simplified state transition diagram of nodes in the broadcast phase is shown in Figure 3(a). The sender after successfully received the broadcast message which the destination address is it, it goes to sleep and will be awaken in the corresponding time slot.

\subsection{Data Phase}

SA-MAC will take the data exchange of nodes randomly distributed to the whole data phase. However, the time slots are randomly selected by the nodes, so the related nodes in the scheduled time slot still need to process a lightweight channel contention.

The state transition diagram of nodes in the data phase is shown in Figure 3(b), the sender wakes up to contend channel in schedule time slot, which uses CW adaptation algorithm like the broadcast phase. During the back-off process, it doesn't immediately go to sleep but to judge if it has listened the RTS which from other nodes. It remains idle listening if the remaining time of current time slot is enough to complete a data exchange. It directly sends RTS to reserve channel if the channel is still idle after $T W C$ timeout. Otherwise, it directly goes to sleep. It goes to sleep after it successfully sends a data packet, regardless of whether it receives ACK.

The receiver wakes up in the scheduled time slot to idle listening. A judgment will be performed if RTS is received from another node. It remains idle listening if the remaining time of current time slot is enough to complete a data exchange. Otherwise, it goes to sleep. During the process of idle listening, if it has listened the RTS which the destination address is it, after sending the corresponding CTS, it goes to sleep regardless of whether the data exchange is successful.

All nodes directly go to the sleep if they have listened CTS from other nodes. 


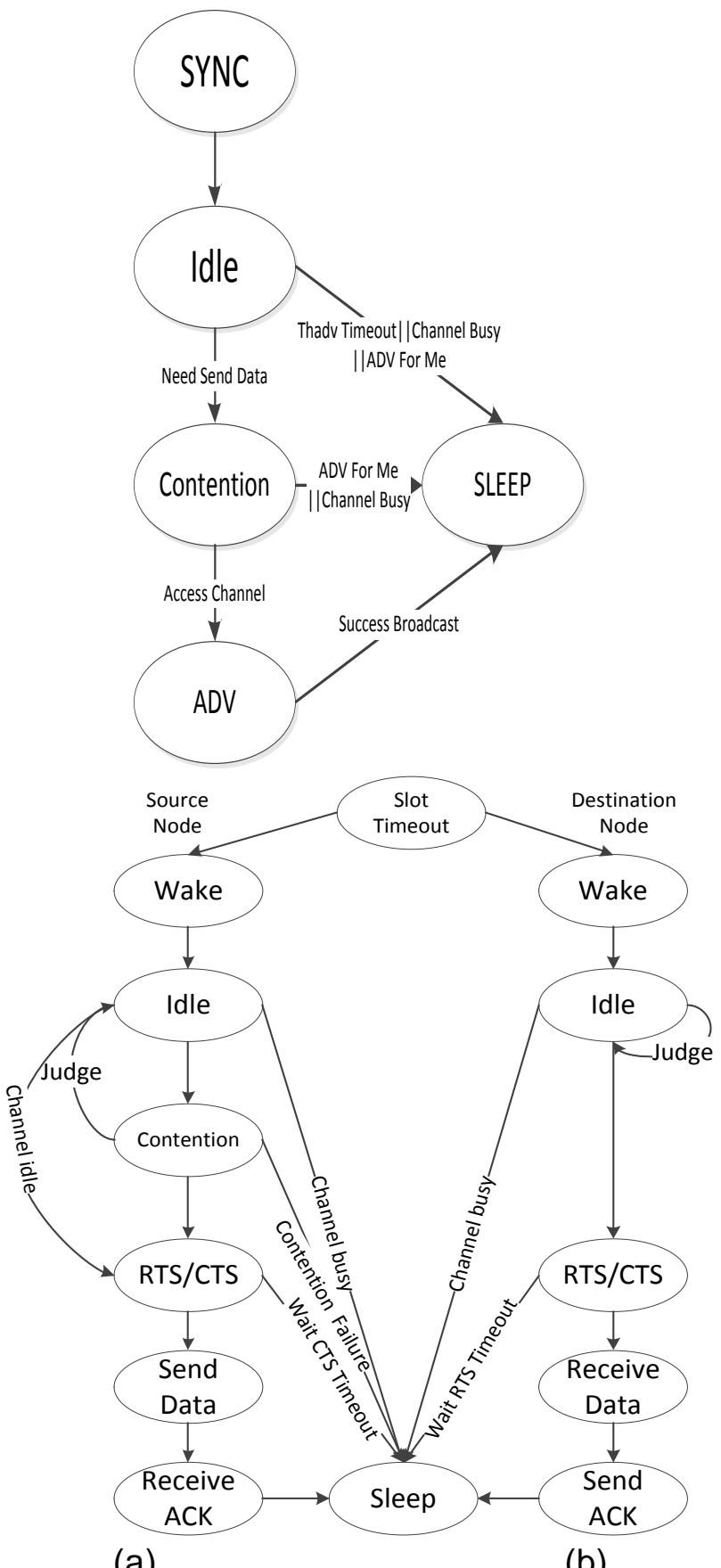

(a)

(b)

Figure 3. Simplified State Transition Diagram of SA-MAC

\subsection{Fast Transmission Mechanism}

Considering the characteristics of WSN business applications with sudden, once nodes from a regional network suddenly monitor large amounts of data packets, there will be a large number of data packets discarded due to the limited data buffer. So, according to real-time usage of the data buffer, we design a fast transmission mechanism (FTS). Nodes use two kinds of transmission mode based on the relationship between $B S$ and $B S T$. Nodes use the normal transmission (NT) when $B S$ is less than or equal to $B S T$. Otherwise, the nodes use FTS.

The working mechanism of FTS is shown in Figure 4. The sender contends channel with the minimum CW to send a control frame, which is defined as MORE_RTS, to 
schedule time slots in the broadcast phase. However, continuous data sending of the nodes will not only interfere with the synchronization phase of next period, but also can interfere with the selection of time slots to other nodes. FTS will reserve time slot number, not the specific time and the maximum time slot number is $m$. Considering that data buffer of the receivers is also limited, the sender should not send too much amount of data packets. It rules that the node goes to the NT when $B S$ decreases to $60 \%$ of $B S T$. The receiver sends a corresponding control frame, which is defined as MORE_CTS, when it receives a MORE_RTS. They go to sleep after they completely exchange control frames.

After enter into the data phase, the sender and the receiver wake up and continuously exchange data packets, using ACK to confirm each data packet and setting the data packet retransmission limit, until both nodes reach the scheduled time slot number. The sender and the receiver give up the data exchange and go to sleep if the remaining time is not enough to complete a data exchange, due to the virtual clusters realize time synchronization and the nodes don't need to send the confirmation message to stop data exchange. Other senders need to judge according to MORE_RTS/MORE_CTS, if the source address and it belong to the same virtual cluster. It judges the control frame which contains time slot number, which is defined as $C$, if $C$ is less than NBmax. It temporarily generates a random number between $[C+1, N B \max ]$, otherwise it directly goes to sleep. It directly goes to sleep until the current period ending if the source address and it don't belong to the same virtual cluster.

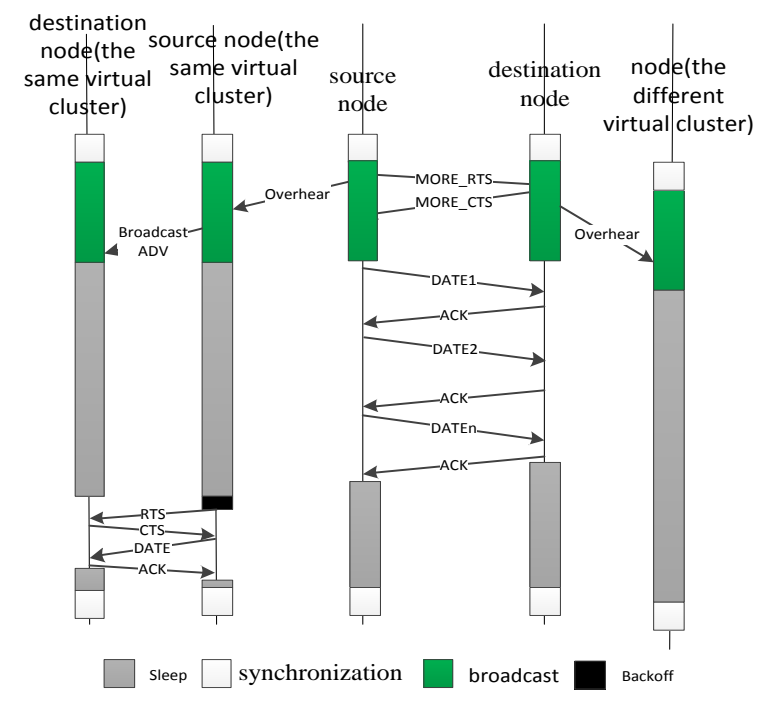

Figure 4. Fast Transmission Mechanism

\section{Analytical Model of SA-MAC}

In this section, we analyze the packet delivery ratio, delay, energy consumption and so on in theory.

\subsection{Packet Delivery Ratio}

This paper mainly uses the time slot reservation mechanism to reduce the probability of data collision, so we analyze the packet delivery ratio of SA-MAC by the time slot reservation mechanism.

Assuming all sensor nodes are within the network and the senders are always able to successfully access the channel in the broadcast phase, the network's data transmission failure will mainly occur in the data phase. This paper uses random variable: $a(t)$ to represent the value of $N b c u r, b(t)$ the number of the remaining time slots when the node 
wakes up at moment $t$, time length of $\mathrm{t}$ is equal to $T_{D}$.so as to construct a two-dimensional discrete time Markov chain: $\{a(t), b(t)\}$.

The remaining parameters are defined as follows:

$k$ : the number of the remaining time slots to send current data packet;

$m$ : maximum number of $N B c u r$, we predict the maximum number of time slot;

$n$ : minimum number of $N B c u r, n=1$.

We define the probability of data transmission failure $P$ : when nodes density reach a certain level and the network is saturated (there are data packets to be sent in data queue), $P$ is a constant in theory. In this paper, we make a model about time slot reservation mechanism, as shown in Figure 5. In the Markov chain, the states of nodes are defined by pairs of integers $\{a(t), b(t)\}$ as follows.

Before data packet is sent:

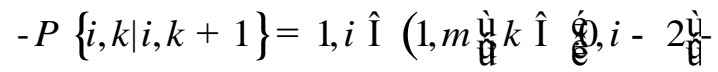

$P\{i, k \mid i, k+1\}$ represents the transition probability from the state $\{a(t)=i, b(t)=k+1\}$ to the state $\{a(t+1)=i, b(t+1)=k\}$, formula 1 represents that the random timer minus 1 in the beginning of moment $t$.

After data packet is sent:

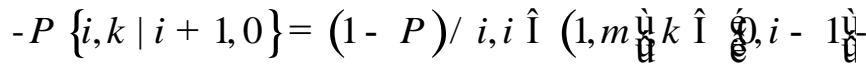

$$
\begin{aligned}
& \text { - } P\{i, k \mid i-1,0\}=P / i, i \text { Î } \\
& \text { - } P\{m, k \mid m, 0\}=P / m, k \text { Î } \\
& \text { - } P\{1,0 \mid 1,0\}=(1-P), k=0 \text { - }
\end{aligned}
$$

Formula 2 represents that after a successful data transmission, NBcur minus $1, N B$ randomly selects from $[1, i]$. Formula 3 represents that after a failing data transmission, $N B$ cur plus $1, N B$ is selected from $[1, i]$ randomly. Formula 4 represents that after a data transmission failure while $N B c u r$ reaching the maximum value, $N B$ is selected from $[1, m]$ randomly. Formula 5 represents that after a successful data transmission while Nbcur reaching the minimum value, $N B$ can only be 1 .

By the ergodicity of Markov chain, we know the stationary distribution of $\{a(t), b(t)\}$ is the limit distribution, so we define the limit distribution:

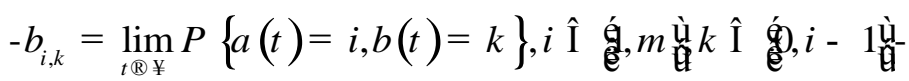

Formula 6 is the limit distribution of $\{a(t), b(t)\}$, so $b_{i, 0}$ has the following properties:

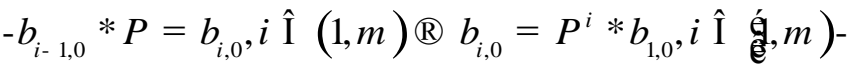

$$
\begin{aligned}
& -b_{m-1,0} * P=(1-P) * b_{m, 0}{ }^{\circledR} b_{m, 0}=\frac{P^{m}}{1-P} * b_{1,0}= \\
& -\stackrel{\mathrm{a}}{i=1}_{i, 0}^{m} b_{i, 0}=\frac{1}{1-P} * b_{1,0}
\end{aligned}
$$

According to the regularity of the Markov chain, we get $b_{i, k}$ :

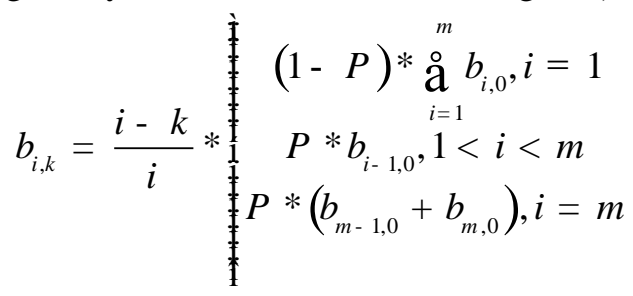

Put the formula 7-9 into the formula 10: 


$$
b_{i, k}=\frac{i-k}{i} * b_{i, 0}, i \hat{\mathrm{I}}(1, m), k \hat{\mathrm{I}}(0, i-1)
$$

Integrated 7-11, all $b_{i, k}$ is represented by $b_{1,0}$ and $P$ :

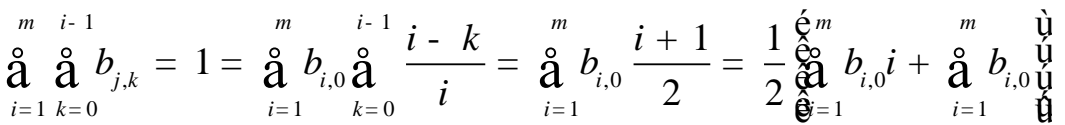

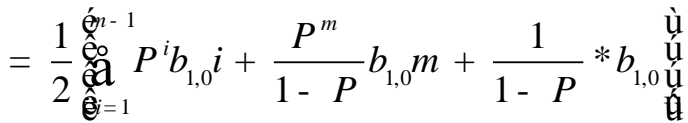

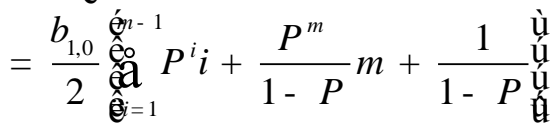

By formula 12:

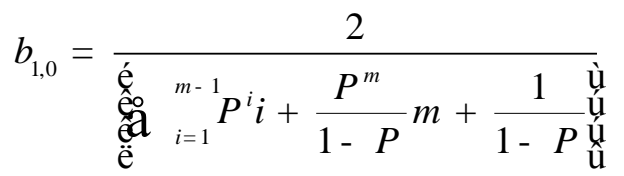

We define the probability of successful data transmission $q$ : assuming all the senders can successfully access channel in the broadcast phase, when network is stable, $q$ equals to the sum of probabilities of successful data transmission of the nodes in each time slot.

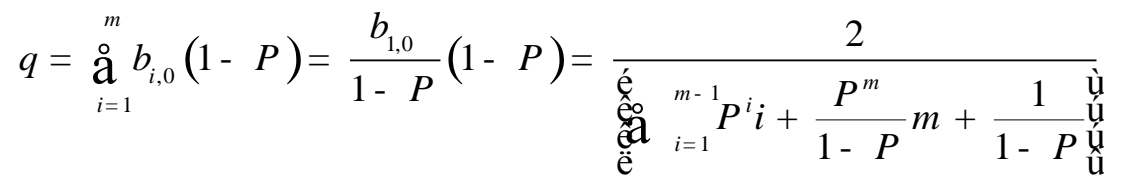

In this case, if there are $w$ nodes in the network, the probability of data transmission failure is equal to the probability of data transmission success of the remaining $(w / m)-1$ nodes, within which at least one node is probable to successfully send the data in corresponding time slot, then $P$ :

$$
P=1-(1-q)^{\frac{w}{n}-1}
$$

So the packet delivery ratio of SA-MAC is $1-P$.

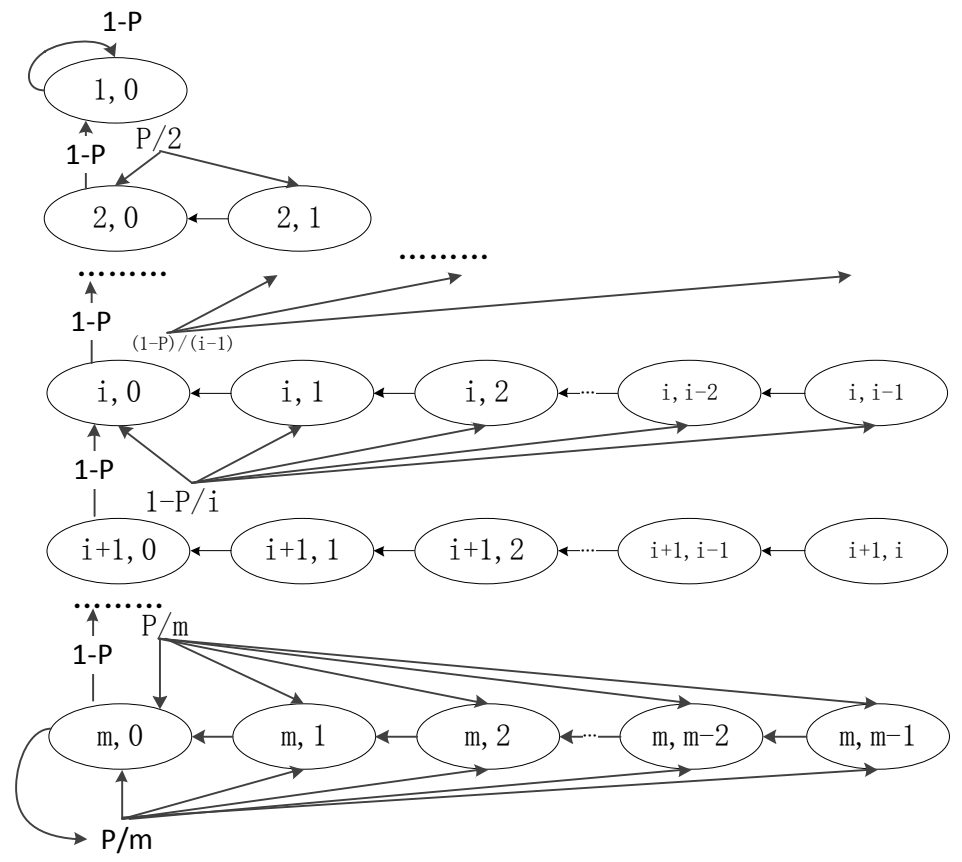

Figure 5. Markov Model of Time Slot Reservation Mechanism 


\subsection{Delay}

Due to the application correlation of WSN, the end-to-end delay is an important indicator to analyze MAC protocols. We will analyze end-to-end delay in theory in this section.

In this section, we set the period length of SA-MAC is a constant, so $m$ is also a constant. In the ideal condition, the delay is calculated:

$$
T_{\text {delay- } S A}=T_{\text {sync }}+T_{a d v}+\frac{{\stackrel{\circ}{{ }_{n=1}^{m}}}_{m}^{m}}{m}+T_{\text {data }}=T_{\text {sync }}+T_{a d v}+T_{D}+T_{\text {data }}
$$

Wherein, $T_{\text {sync }}$ is the time of status update and synchronization in the synchronization phase, which, under normal circumstance, is $T_{s} . T_{a d v}$ is the time needed by the nodes to schedule time slot in the broadcast phase, including back-off time, idle listening time and time of transmit broadcast messages. Under normal circumstance, the maximum value is $T_{A} . T_{\text {data }}$ is the work time needed by the nodes to be activated in corresponding time slot in the data phase, including idle listening time, send and receive data packets and back-off time. Under normal circumstance, the maximum value of $T_{\text {data }}$ is $T_{D}$.

We further assume that the data packet is ultimately transmitted successfully while the network is saturated, then we analyze delay as shown in Formula 17, where $k$ is the upper limit of the packet retransmission, $T_{\text {cycle }}$ is the time length of the period.

$$
\begin{aligned}
& T_{\text {delay }}=(1-P) T_{\text {delay-SA }}+P(1-P)\left(T_{\text {cycle }}+T_{\text {delay- } S A}\right)+\ldots \\
& +P^{k}(1-P)\left(k T_{\text {cycle }}+T_{\text {delay }-S A}\right)=
\end{aligned}
$$

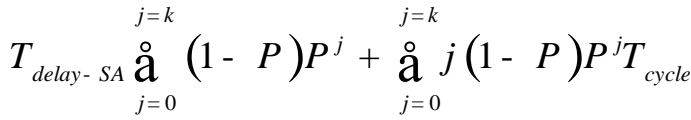

\subsection{Energy}

The related scholars usually use idle listening time of nodes to measure the irrespective energy consumption of MAC protocols. This paper also uses it to measure the energy consumption of SA-MAC.

In the ideal condition, we analyze the listening time of SA-MAC in Formula 18.

$$
\text { Time }^{\text {ideal }}=T_{\text {sync }}+T_{\text {adv }}+T_{\text {data }}
$$

When network load is light, there is $T_{\text {data }}=0, T_{a d v}=T h a d v$. Therefore, the listening time:

$$
\text { Time }^{\text {light }}=T_{\text {sync }}+\text { Thadv }
$$

When the network is saturated, we further analyze the listening time of SA-MAC, as shown in Formula 20, where $T_{\text {back-off }}$ is the time of failure to contend channel in data phase.

$$
\begin{aligned}
& \text { Time } e^{\text {saturated }}=(1-P) \text { Time }^{\text {ideal }}+P(1-P)\left(T_{\text {sync }}+T_{\text {adv }}+T_{\text {back-off }}+\text { Time } e^{\text {ideal }}\right) \\
& +\ldots+P^{k}(1-P)\left(k\left(T_{\text {sync }}+T_{\text {adv }}+T_{\text {back-off }}\right)+\text { Time }^{\text {ideal }}\right)
\end{aligned}
$$

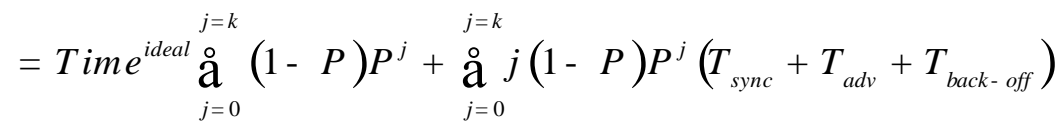




\section{Simulation Results}

In this paper, we use network simulation software NS2 to simulate SA-MAC3 ( $m$ is 3 ) and SA-MAC5's ( $m$ is 5) performance respectively in single-hop and multi-hop scenario, and compare them with ADV-MAC, S-MAC10 (10\% duty-cycle), S-MAC20 (20\% dutycycle). The main parameters of the simulation experiment are shown in Table 1.

Table 1. Partial Simulation Parameter Setting

\begin{tabular}{ll}
\hline parameter & value \\
\hline transmission distance & $250 \mathrm{~m}$ \\
data packet length & $50 \mathrm{Byte}$ \\
simulation time & $1000 \mathrm{~s}$ \\
routing & $\mathrm{DSR}$ \\
sending power & $22.6 \mathrm{mw}$ \\
received power & $15.1 \mathrm{mw}$ \\
listening power & $15 \mathrm{mw}$ \\
sleeping power & $0.5 \mathrm{mw}$ \\
\hline
\end{tabular}

\subsection{Simulation Results in Single-hop Scenario}

This paper assumes that 20 nodes are placed within the scope of $150 \mathrm{~m} * 150 \mathrm{~m}$, so that all nodes are in transmission range of each other, including five source nodes in the area. Within all the nodes, one node is selected as test node, the other nodes as interfering nodes.

In this paper, all the packet arrival interval of the interfering nodes is set to $5 \mathrm{~s}$, and we simulate the performance of protocols when the packet arrival interval of the test node is increased from $1 \mathrm{~s}$ to $10 \mathrm{~s}$. Figure 6 (a) shows the variation of packet delivery ratio. When packet arrival interval is low, packet delivery ratio of SA-MAC is always better than the other protocols, because SA-MAC reduces the probability of data collision. With the increase of packet arrival interval, network load is gradually lower, the packet delivery ratio of all protocols ultimately reach a constant level. Figure 7(a) shows the variation of delay. The simulation result shows that the delay of SA-MAC is significantly less than other protocols because the SA-MAC nodes can send more data packets than other protocols in a work period. Figure 8(a) shows the variation of total average energy consumption. We observe that the average energy consumption of SA-MAC is always at a lower level because SA-MAC makes the nodes, which do not need to exchange data, in a state of sleep and uses low duty ratio mechanism to reduce the idle listening time.

From the above figures, it is found that the energy consumption of SA-MAC5 is 10$19 \%$ less when compared to SA-MAC3, 15-21\% less when compared to ADV-MAC, 33$45 \%$ and $50-65 \%$ less when compared to S-MAC10 and S-MAC20. As is shown from the above, SA-MAC reduces the energy consumption while improving the packet delivery ratio and delay.

\subsection{Simulation Results in Multi-hop Scenario}

This paper assumes that 60 nodes are placed within the scope of $600 \mathrm{~m} * 600 \mathrm{~m}$, including ten source nodes in the area, like single-hop scenario. One node is selected as test node and the other nodes as interfering nodes.

Figure 6(b) shows the variation of the packet delivery ratio, with the increase of the packet arrival interval, packet delivery ratio of SA-MAC is maintained in a controllable range, and better than other MAC protocols. Figure 7(b) shows the variation of delay, the 
delay of SA-MAC is better than other protocols. Figure 8(b) shows the variation of total energy consumption averagely, we observe that the average energy consumption of SAMAC is less than other protocols, and the variation range is smaller.

It is seen that SA-MAC5 achieves 12-19\% less energy consumption compared with SA-MAC3. Compared with ADV-MAC, the energy consumption is reduced by $14-22 \%$, and compared to S-MAC10 and S-MAC20, the energy consumptions are reduced by 32$44 \%$ and 51-69\%, respectively. As seen from figure 6(b) and 7(b), these energy savings are obtained without compromising the packet delivery ratio and delay.

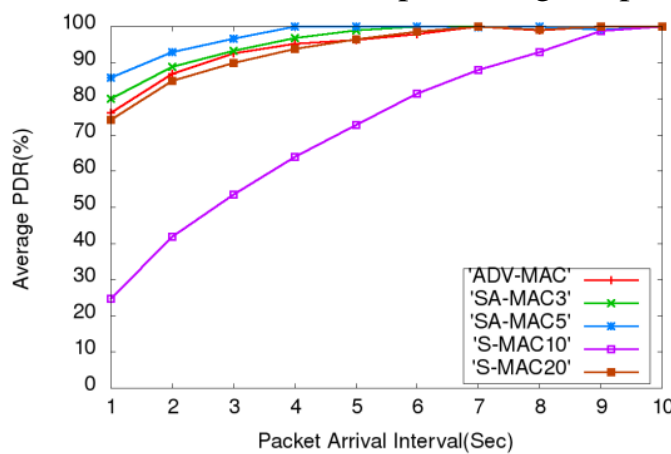

(a)

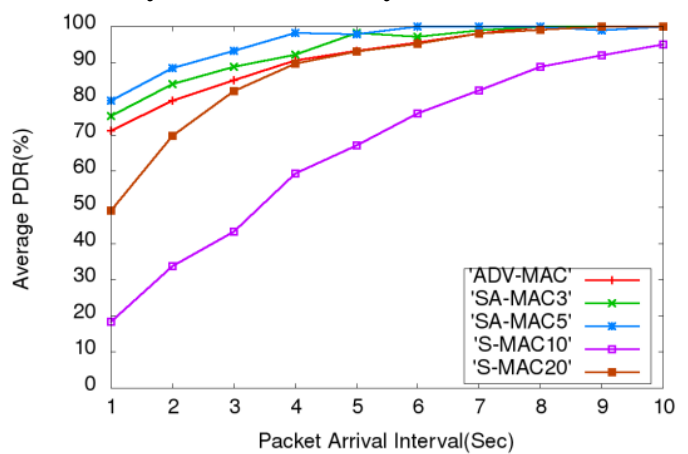

(b)

Figure 6. Packet Delivery Ratio

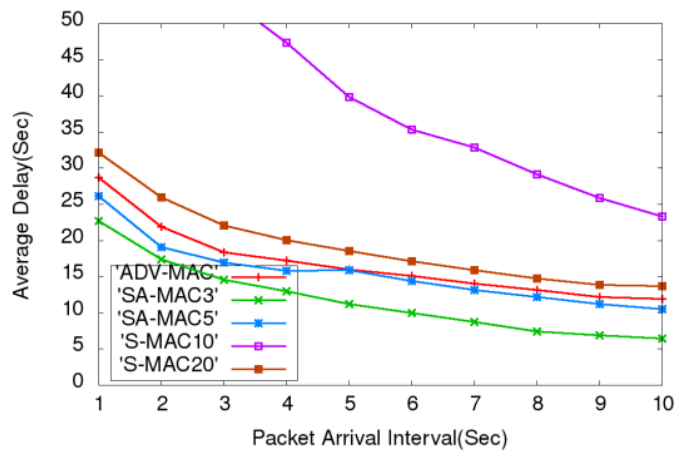

(a)

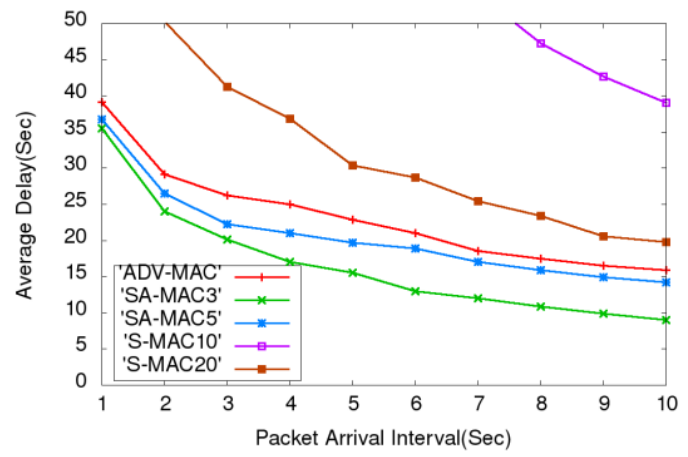

(b)

Figure 7. Average Delay

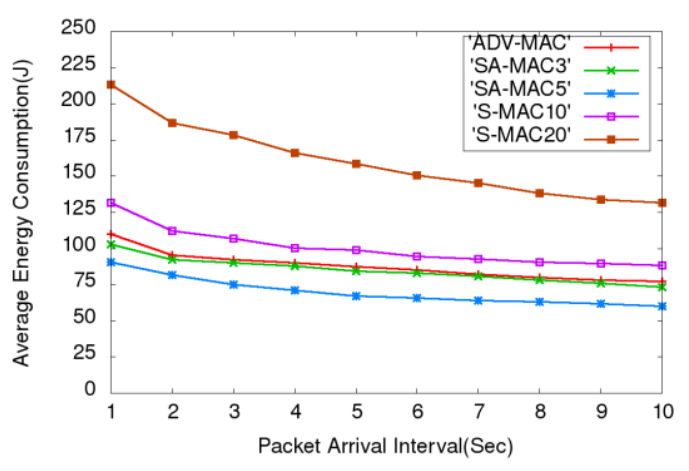

(a)

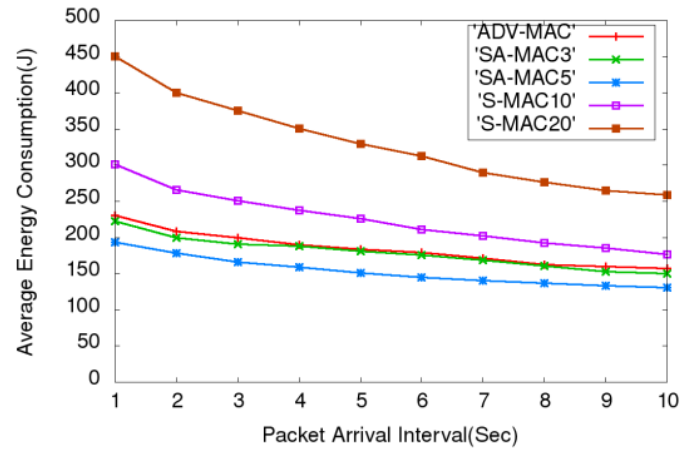

(b)

Figure 8. Average Energy Consumption 


\section{Conclusion}

This paper proposes a competitive MAC protocol, SA-MAC, which is based on broadcast messages. The protocol uses time slot selection algorithm, CW adaptation algorithm and fast transmission mechanism to make SA-MAC has the advantages of low collision, low power consumption and adaptation. Simulation results show that, compared with the S-MAC and ADV-MAC, SA-MAC has a better performance on packet delivery ratio, delay and energy consumption whether in the single-hop or multi-hop scenario. In addition, the performance of SA-MAC is different when the time slots' number $m$ varies. SA-MAC ( $m$ is 3 ) has a relatively better performance when the network load is light, while SA-MAC ( $m$ is 5 ) is a good choice when the network load is heavy.

\section{Acknowledgments}

This work is supported by the National Basic Research Program of China (No. 2014CB049401), the Project of Basic and Advanced Technology Research of Henan Province of China (No. 132300410001), and the Program for Innovative Research Team (in Science and Technology) in University of Henan Province (No. 15IRTSTHN008).

\section{References}

[1] F. Akyildiz, W. Su and Y. Sankarasubramaniam, "Wireless Sensor Networks: a Survey", Computer Networks, vol. 38, no. 4, (2002), pp. 393-422.

[2] M. Kumar, K. Pandey and M. M. Sharma, 'Survey on wireless sensor networks using MAC protocol'. Proceedings of the 9th IEEE International Conference on Industrial and Information Systems, Gwalior, India, (2014), pp. 1-6.

[3] I. Jang, D. Pyeon and S. Kim, "A Survey on Communication Protocols for Wireless Sensor Networks", Journal of Computing Science \& Engineering, vol. 7, no. 4, (2013), pp. 231-241.

[4] S. C. Kim, J. H. Jeon and J. J. Kim, "Energy and delay efficient duty-cycle MAC protocol for multi-hop wireless sensor networks", International Journal of Multimedia and Ubiquitous Engineering, vol. 10, no. 2, (2015), pp. 361-370.

[5] H. Lee, K. Chung and K. Jhang, "TDMA implementation for simultaneous signal acquisition of machine condition in wireless sensor networks", IEEE Communications Magazine, vol. 22, no. 11, (2014), pp. 54-59.

[6] Z. H. Chen and A. Khokhar, "Self organization and energy efficient TDMA MAC protocol by wake up for wireless sensor networks", Proceeding of the Sensor and Ad Hoc Communications and Networks, (2004).

[7] J. Li, W. Zeng and A. Arora, "The configuration space of duty-cycled CSMA-based wireless MACs", Wireless Networks, vol. 20, no. 8, (2014), pp. 2561-2579.

[8] F. Chungjung, A. Lee, M. Jin and K. Chengyan, "A latency MAC protocol for wireless sensor networks", International Journal of Future Generation Communication and Networking, vol. 2, no. 1, (2009), pp. 41-54.

[9] W. Ye, J. Heidemann and D. Estrin, "An energy-efficient MAC protocol for wireless sensor networks", Acm Infocom, vol. 3, no. 10, (2002), pp. 1567-1576.

[10] W. Ye, J. Heidemann and D. Estrin, "Medium Access Control With Coordinated Adaptive Sleeping for Wireless Sensor Networks", In IEEE Trans. on Networking, vol. 3, no. 12, (2004), pp. 493-506.

[11] I. Rhee, A. Warrier and M. Aia, "Z-MAC: A Hybrid MAC for Wireless Sensor Networks", Networking IEEE/ACM Transactions, vol. 16, no. 3, (2005), pp. 90-101.

[12] J. Polastre, J. Hill and D. Culler, "Versatile low power media access for wireless sensor networks", Proceedings of the 2nd international conference on Embedded networked sensor systems ACM, (2004), pp. 95-107.

[13] K. Sohrabi, V. Ailawadhi and G. Pottie, "Protocols for Self-Organization of Wireless Sensor Networks", IEEE Personal Communications Magazine, vol. 7, no. 10, (2000), pp. 16-27.

[14] W. Fang, Q. Zhou, Wang Z and Q. Liu, "An Adaptive Transmission Scheme for Wireless Sensor Networks", International Journal of Future Generation Communication and Networking, vol. 6, no. 1, (2013), pp. 35-44.

[15] S. Ray, I. Demirkol and W. Heinzelman, "ADV-MAC: Advertisement-based MAC Protocol for Wireless Sensor Networks", Proceedings of the 5th International Conference on Mobile Ad-hoc and Sensor Networks, (2009), pp. 1-2.

[16] S. Ray, I. Demirkol and W. Heinzelman, "ADV-MAC: Analysis and Optimization of Energy Efficiency through Advertisements for Wireless Sensor Networks", Elsevier Ad Hoc Networks Journal, vol. 9, no. 5, (2011), pp. 876-892. 


\section{Authors}

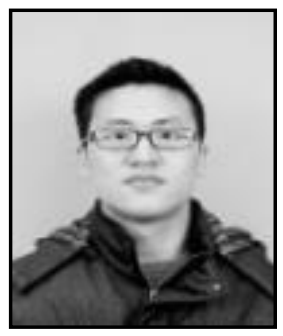

Bingwu Kang, he received the B.S. degree in Luoyang Normal University, Luoyang, China in 2013. He is currently working towards M.S. degree in Henan University of Science and Technology, Luoyang, China. His research interests include WSNs and MAC.

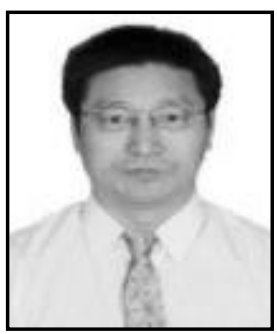

Guoqiang Zheng, he received the Ph.D. degree in Communication and information systems professional from Xi'an Jiaotong University, China, 2008. He is a professor at Henan University of Science and Technology on College of Electronic Information Engineering, China. His research interests include wireless communication technology, network communication protocol and software radio theory.

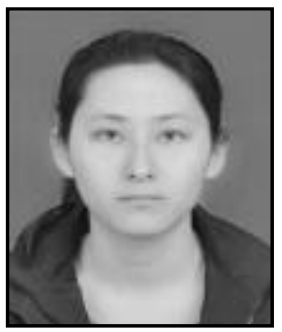

Fangge Nie, she received the B.S. degree in Henan University of Science and Technology, Luoyang, China in 2012. She is currently working towards M.S. degree in Henan University of Science and Technology, Luoyang, China. Her research interests include WSNs and Opportunistic Routing.

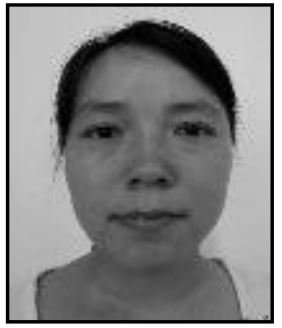

Huahong Ma, she received her master degree in Signal and Information Processing in July 2005 at Yunnan University, China. Now, she is a Ph.D. candidate in Control Science and Engineering at Henan University of Science and Technology. Her main research interests are Crowd Sensing Network and Internet of Things.

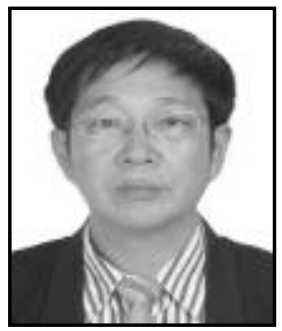

Jishun Li, he received the Ph.D. degree in Mechanical Manufacture and Automation from Shanghai Jiaotong University, China, 1996. He is a professor at Henan University of Science and Technology on College of Mechatronics Engineering, China.

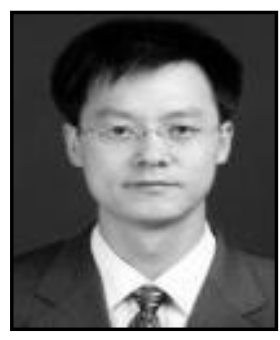

Yujun Xue, he received the Ph.D. degree in mechanical design and theory from Shanghai Jiaotong University, China, 2002. He is a professor at Henan University of Science and Technology on College of Mechatronics Engineering, China. 


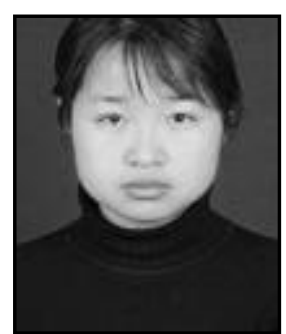

Peipei Li, she received the M.S. degree in Communication and Information System in Nanjing University of Science and Technology, Nanjing, China in 2006. She is a lecturer at Henan University of Science and Technology on College of Electronic Information Engineering, China. 